\title{
Efficacy of Diabetes-Specific Formulas as Meal Replacements in Diabetic Patients: An Overview
}

\author{
Anayanet Jáquez*, José J Sánchez, Noemí Acevedo, and Elizabeth Guzmán
}

Pontificia Universidad Católica Madre y Maestra, Dominican Republic

*Corresponding author: Anayanet Jáquez, MSc, MD, MRes, Pontificia Universidad Católica Madre y Maestra (PUCMM), Dominican Republic, Tel: 8093066405; Email: drajaqueznc@gmail.com

Received: 06 Apr, 2020 | Accepted: 29 Apr, 2020 | Published: 08 May, 2020

Citation: Jáquez A, Sánchez JJ, Acevedo N, Guzmán E (2020) Efficacy of Diabetes-Specific Formulas as Meal Replacements in Diabetic Patients: An Overview. Int J Endocrinol Metab Disord 6(2): dx.doi.org/10.16966/2380-548X.168

Copyright: (C) 2020 Jáquez A, et al. This is an open-access article distributed under the terms of the Creative Commons Attribution License, which permits unrestricted use, distribution, and reproduction in any medium, provided the original author and source are credited.

\begin{abstract}
Diabetes-specific formulas have shown to be effective at improving glucose control with additional nutritional benefits. The aim of this review is to assess the current knowledge on the different types of DSF (Diabetes-Specific Formulas) and how they affect the weight, HbA1c (glycosylated hemoglobin), glucose, insulin and lipid profiles. Database research was made with diabetes and nutritional formulas as keywords. From over 60,000 titles retrieved from 2005 to 2020, only 34 were chosen based mainly on their methodology and results. Results show that for glycemia control, high fiber carbohydrates are more effective as well as high protein formulas. For HbA1c, oat seemed to be less effective than Meal Replacement (MR) and a protein-rich formula proved to be effective in the long-term. One of the most researched benefits in MR plans is weight loss, evidences show up to a $5-10 \%$ decrease in interventions with DSF formulas. Other advantages of this approach include greater glycemic control, insulin sensitivity and lower postprandial secretion, which consequently lead to a decrease in morbidity and mortality associated with cardiovascular causes. About lipid profile values; HDL, LDL and Total cholesterol, existing evidence differ from one another, so it is necessary to investigate further to reach a consensus.
\end{abstract}

\section{Introduction}

Worldwide prevalence of diabetes has increased over the last 40 years from $4.7 \%$ to $8.5 \%$ of the adult population, especially those in 2016, around 1.6 million deaths occurred as a direct consequence of diabetes, making it the $7^{\text {th }}$ leading cause of death in that same year [1]. Poor glycemic control and dyslipidemia are very important aspects of the type 2 diabetic patient follow up, mainly because of the systemic consequences high blood glucose and dyslipidemia have proven to promote [2]. Many comorbidities such as hypertension, which affects at least $65 \%$ of diabetic patients, is also linked to other fatalities like coronary artery disease, myocardial infarction, stroke and congestive heart failure [3].

Additionally, microvascular complications as neuropathy, retinopathy and nephropathy play an important role in advanced diabetes mellitus. This is all possible because hyperglycemia promotes irregularities in the protein kinase $\mathrm{C}$, polyol and hexosamine pathways, along with the free radical damage from Reactive Oxygen Species (ROS) and glycated end products which can produce endothelial dysfunction. Because of all this, the therapeutic target of diabetes is to keep blood glucose levels as low and as stable as possible, so that damage can be partially, if not completely, prevented [4]. DSF formulas have shown to help maintain glucose and lipid profiles at adequate levels when used for short periods of time [5].
Nevertheless, not all DSF formulas are created equal, nor is their effectiveness at short and long term. That is why the aim of this review is to give an overlook of the effects of DSF formulas (as meal replacements) on blood glucose, insulin, HbAlc, lipid profile and weight of these patients [6].

\section{Methods}

\section{Search terms}

Databases used for searching titles included Google Scholar, PubMed, SciELo and Ebsco. Keywords or search terms varied among the phrases: "diabetes and polymeric formulas", "diabetes and nutritional formulas and "diabetes and nutrition". Filters were applied when possible, such as "humans". Year range went from 2005 to 2020, English and Spanish language articles were allowed only.

\section{Selection criteria}

Inclusion criteria for the articles included the following: those studies related to humans, English and Spanish language publications, relevant results for the aim of this review, full-text article availability. On the other hand, texts with redundant results, different target populations, tube feeding, diet plans, poor methodology and grey literature were not considered for the writing of this review [Figure 1]. 


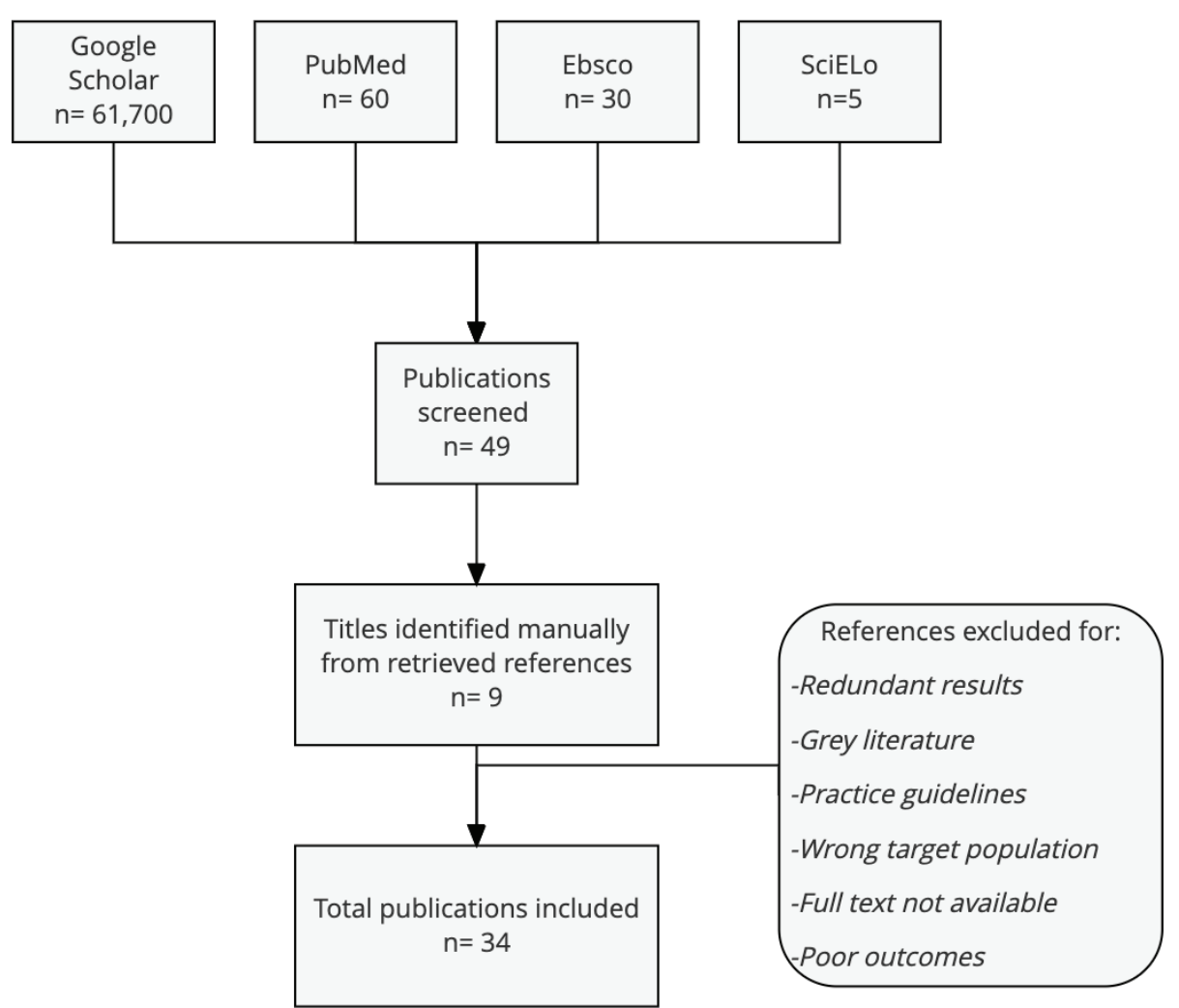

Figure 1: Flow chart of the review literature.

\section{Results and Discussion}

\section{Effect of meal replacements on blood glucose}

When replacing one meal, a lower glycemic index has been linked with the consumption of DSF formulas with extended-release carbohydrates, if compared with the same amount of carbohydrates from a reference food, such as bread or glucose. This means that a lower glycemia was observed in subjects consuming DSF formulas $[7,8]$. Something similar happened when patients were given an oral nutritional formula based on a protein blend, fiber and a fat blend high on oleic oil. Researchers compared it with those receiving a cornflakes and milk isocaloric meal and found that the area under the curve for post-meal glycemia was significantly lower in the experimental group at $30 \mathrm{~min}(\mathrm{p}=0.003), 60 \mathrm{~min}(\mathrm{p}=0.0001), 120 \mathrm{~min}(\mathrm{p}=0.0001)$, and 180 $\min (\mathrm{p} 0.0001)$ [9].

Likewise, postprandial glycemia was also significantly lower and got back to baseline faster when experimenting with two DSF formulas versus an isocaloric amount of oatmeal for breakfast. This could be explained by the low glycemic index of the formulas and macronutrient composition [10]. Another study compared two DSF formulas vs. a standard control formula for a two-year follow-up period, results in plasma glucose levels showed that compared with the standard control formula, both DSF lowered insulin requirements, improving capillary glycemia $(146.1 \pm 45.8 \mathrm{mg} / \mathrm{dL}, \mathrm{p}<0.001)$ [11].

Taking a high-energy, high-protein DSF formula and substituting the maltodextrin for isomaltose from an oral nutrition supplement resulted in an attenuated postprandial glucose level. However, postprandial peak glucose concentration in both formulas did not differ significantly ( $p=0.107)$ [12]. Another hyper-protein but very lowcarbohydrate nutrition formula was tested against a standard protein formula. The first one showed to keep blood glucose concentrations within very small variations when followed up from 30 to $150 \mathrm{~min}$. These could be very helpful in malnourished patients suffering from muscle wasting or sarcopenia, given the fact that most DSF formulas tend have a higher lipid content and normoprotein distribution [13].

A higher fat and fiber content, as well as lower carbohydrate and the presence of fructose in DSF oral nutritional formulas has shown to contribute to a significantly lower glycemic index and glycemic control when compared to standard nutritional formulas. Although, it is important to note that a high-fat content meal could promote weight gain and lipid distortions among diabetic patients despite of its effects on blood glucose. In the Look AHEAD study, findings showed significant reduction in weight and blood glucose levels on those type2 diabetes patients under MR plans with DSF in comparison to others carrying on standard diets with equal caloric count [14].

\section{Effect of meal replacements on HbAlc}

When diabetic patients were given a high fiber meal replacement for breakfast containing rice, soybean, resistant starch and oat dietary based meal, was measured as a long-term glycemic control variable. For patients who took the oat-based meal, HbAlc increased by $0.3 \%$ ( $95 \% \mathrm{CI}, 0.1 \%$ to $0.5 \%, \mathrm{p}=0.005$ ). In contrast to those who took the high fiber breakfast replacement, whose HbAlc diminished by 
$-0.2 \%$ (95\% CI, $-0.38 \%$ to $-0.07 \%, \mathrm{p}=0.004)$. This suggests further consideration when recommending oat-based meals for the long term to these patients [15].

On the other hand, in this 12 -week trial, patients replaced three meals a day with a protein-rich formula during week 1 . In weeks 2-4, both dinner and breakfast were replaced and finally from week 5 through 12 , only dinners were exchanged. After 8 weeks, HbAlc decreased from initially $8.8 \%$ to $7.7 \%(1.1 \%)(\mathrm{p}=0.002)$. At the end of the study there was a slight increase of HbAlc to $8.1 \%$, which was still statistically significantly lower than the reference $[0.8 \%$ $(1.4 \%) ; \mathrm{p}=0.048]$, proving the effectiveness of the protein-rich meal replacement formula in long term control of glycemia [16].

An intervention consisting of a personalized diet, regular motivation and meal replacement with a low carbohydrate formula was able to decrease $\mathrm{HbAl} 1 \mathrm{c}$ to a mean of [95\% confidence interval] $-0.97 \%$ [-1.21 to -0.74$]$ just as the previous studies demonstrated. Nevertheless, it is difficult to point out which of the elements of the intervention contributed the most to the lowering of $\mathrm{HbAlc}$, which could diminish the clinical significance of the results from this study [17]. Other data demonstrate that three-month interventions following a 1 or 2 meal replacements daily program lead to a great decrease $(0.11 \%)$ in $\mathrm{HbAlc}$ [18]. In the Look AHEAD trial study, individuals exposed to the intervention had better odds to lost $>10 \%$ of their initial weight after the 1-year period of follow up, at the same time, this group also got lower results in $\mathrm{HbAlc}(6.1 \% \pm 0.7$ men, $6.3 \% \pm 1.0$ women $)$ compared to their baseline data $(7.0 \% \pm 1.1) ; \mathrm{p}(<0.0001)$ [19].

\section{Effect of meal replacements on weight}

Different studies have shown that weight loss of approximately $5-7 \%$ is a determining factor in reducing the mortality rate due to cardiovascular reasons; sleep apnea; female sexual dysfunction; hospital stay and urinary symptoms in overweight patients with typess 2 diabetes [20].

The Look AHEAD initiative, focused on Intensive Lifestyle Intervention (ILI), consists of a program that integrates Meal Replacements (MR) with physical activity, and its results reported that after 1 year the experimental group participants lost approximately 8.6 $\mathrm{kg}(\mathrm{P}<0.001)$ compared to the control group that followed a dietary scheme with the same amount of calories per day. The most relevant data showed a positive correlation between weight loss and the quantity of meals replace by a nutritional formula per day $(\mathrm{r}=0.32, \mathrm{p}<0.001)$, also, likewise data analysis demonstrated that same candidates were 4 times more likely to achieve a weight loss greater than $10 \%$ at the end of the study. Finally, the consumption of meal replacements was the third most influential factor to reach the goal of weight loss, followed by greater self-reported physical activity [21].

Interventions such as; Slim-Fast and Weight Watchers (both dietary plans calculated by energy intake for achieving weight loss), low carb diet and combined diet- exercise plan; have demonstrated effectiveness in decreasing some anthropometric measures of participants, specifically the perimeter of waist and weight [22]. Looking into a short MR intervention performed on 2009, diabetic patients (BMI 33$44 \mathrm{~kg} / \mathrm{m}^{2}$ ), were randomized in counseling sessions and hypocaloric diet $(60 \%)$ reduction of subgroups. Those patients treated with the specific formula Glucerna SR developed a greater and sustained weight loss over time. This formula provides 206 calories per serving distributed in $9 \mathrm{~g}$ protein, $25 \mathrm{~g}$ carbohydrate and 24 other compounds. Specifically, maltodextrin; sunflower oil; soy oil, fructose; minerals (such as potassium, magnesium, sodium, copper, zinc and ferrous sulfate); vitamins B, A, K1, D2, antioxidants, taurine and L-carnitine
[23]. Another study, followed a sample of patients for 8 weeks, where breakfast, lunch and dinner were replaced by a specific liquid formula composed of equal parts of proteins and carbohydrates; and 5\% fat, the final data showed a marked decrease in weight $(-9.6 \mathrm{Kg})$ and percentage of body fat $(-7.6 \%)$ in the participants [24].

The standard Soy-Yoghurt-Honey (SYH) formula known as almased for commercial purposes; is composed mainly by soy and milk protein, in addition to antioxidants, enzymes and oligofructose from honey. In order to quantify its efficacy this formula was implemented during a controlled trial conducted in 88 diabetic and overweight subjects, randomized in two groups; meal replacements formulas and conservative therapy (lifestyle modifications). The high protein formula was administrated for 6 weeks, obtaining better results in terms of weight reduction (180\% more) and fat mass $(\mathrm{p}<0.01)$ in comparison to lifestyle group. With that in mind experts have approved the meal replacement diet approach as an effective strategy to lose weight in diabetic patients, especially if it is part of the initial phase in the therapeutic program [25]. Another soy-based formula showed similar results in a longer intervention, where weight loss in female patients was greater $(-7.6 \pm 7.9 \mathrm{~kg})(\mathrm{p}<0.001)$ than the control group [26].

\section{Effect of meal replacements on fasting insulin}

Commonly used nutritional formulas are composed of high percentages of carbohydrates and/or proteins. Additionally, specific features of DSF include their low glycemic index, normoproteic and normocaloric balance, as well as $100 \%$ PHGG (partially hydrolyzed guar gum) soluble fiber, target population are diabetics who need to maintain this type of diet for long amounts of time. An experimental therapy was carried out in a clinical trial on 15 patients, achieving a lower average in the concentration of serum insulin $(p=0.039)$ compared to the subgroup that took the standard polymeric nutritional formula. Specifically, insulin levels in the 60 and 90 minutes of the curve showed lower insulin requirements [27].

The Soy-Yoghurt-Honey (SYH) formula, was a standard weightloss formula used to replace breakfast in a group of patients during a randomize trial, obtaining post-prandial glycemic and insulinemic responses lower than the traditional breakfast group [25]. Other meal replacement therapies also showed significant decreases in serum insulin levels $(14.1 \pm 1.3 \mathrm{IU} / \mathrm{ml}, \mathrm{p}<0.05)$ compare to $29.3 \pm 10.9 \mathrm{IU} /$ $\mathrm{ml}$ in groups that had other diet programs [24]. Another specific formula with similar effects is Glucerna SR, which was incorporated into a randomized, crossover clinical trial, within 14 obese patients. Upon completion of the intervention, participants showed an increase in insulin sensitivity coupled with a decrease in insulin secretion [28].

When it comes to comparing standard formulas $v s$. Oral Nutritional Supplements for Diabetic patients (ONS-D), the latter demonstrate lower glycemic response and greater control in postprandial appetite. These outcomes are presumably because ONS-D such as Glucerna and Diasip are composed of sucrose analogs; sucromalt and isomaltulose respectively. Both components are low digesting carbohydrates that provoke increase GLP-1 secretion, which consequently might lower GIP and insulin concentration. On the other hand, blood glucose levels after ingesting ONS-D returned to baseline in approximately 150 minutes in contrast to standard nutritional supplements (180 minutes), which proves that ONS-D promotes a healthier metabolic profile in diabetic patients [29].

\section{Effect of meal replacements on lipid profile}

When using nutritional formulas under a properly structured plan, reductions in the biochemical values of cholesterol, triglycerides, LDL 
and HDL are also observed [30]. An example of this was evidenced in a multicenter, controlled trial, where the experimental group of patients received once a day the formula of meal replacement 'Once Pro' composed of $40 \%$ carbohydrate, $20 \%$ protein and $40 \%$ fat added to a controlled diet, and the other subgroup only followed a dietary regimen. At the end of 3 months both groups obtained lower HDL cholesterol values, but only the MR group had lower LDL and total cholesterol values [31]. In another intervention, using the Glucerna formula similar results were obtained, post analysis data showed decreased values in C-HDL-C, VLDL-C and triglycerides [32] [Tables 1 and 2].

\section{Recommendations and Conclusion}

In overweight patients, DSF should be taken 2-3 times per day together with a reduced calorie meal plan, either as a calorie replacement for a meal, as a partial meal or as a snack. The calorie goal for this group of patients is as follows: patients $<250 \mathrm{lb}=1,200$ to 1,500 calories, while patients $>250 \mathrm{lb}=1500$ to 1800 . The approach in normal weight patients varies if the diabetes is controlled ( $\mathrm{HbAlc} \leq 7 \%$ ) or uncontrolled (HbAlc $>7 \%)$. For patients with controlled diabetes, the use of DSF depends on the physician criteria an the patients' characteristics. However, for patients with uncontrolled diabetes, DSF should be incorporated 1-2 times per day into a meal plan, either as a calorie replacement for a meal, as a partial meal or as a snack. In underweight patients, is recommended to use 1-3 units of DSF per day, depending on management goals [33].

Standard formulas have been associated with adverse effects such as hyperglycemia, osmotic diuresis and loss of electrolytes, so they are not ideal for diabetic patients. To cover this sector of the population,

Table 1: Shows distinct diabetes-specific formulas and their composition.

\begin{tabular}{|c|c|c|c|c|}
\hline Formula & Protein & $\mathrm{CHO}$ & Fat & Fiber \\
\hline \multirow{3}{*}{$\begin{array}{l}\text { Extended-release carbohydrates } \\
\text { (Glucerna) [7] }\end{array}$} & Total: $21 \%$ & Total: $56 \%$ & Total: $15 \%$ & \multirow{3}{*}{$3.6 \mathrm{~g} / 100 \mathrm{~mL}$} \\
\hline & -Calcium caseinate (98\%) & $\begin{array}{c}\text { Maltodextrin, fructose and } \\
\text { maltitol }\end{array}$ & Oleic acid and soy oil. & \\
\hline & -Soy protein $(2 \%)$ & & & \\
\hline \multirow{2}{*}{ High protein content (Diasip) [8] } & Total: $16 \%$ & Total: $35 \%$ & Total: $49 \%$ & \multirow{2}{*}{$2.5 \mathrm{~g} / 100 \mathrm{~mL}$} \\
\hline & Protein blend & Fructose $(2.3 \mathrm{~g})$ & -MUFA (3.6 g / 100mL) & \\
\hline \multirow{5}{*}{$\begin{array}{l}\text { High energy and high protein } \\
\text { (DiaCare) [11] }\end{array}$} & Total: $26 \%$ & Total: $41 \%$ & Total: $32 \%$ & \multirow{5}{*}{$0.7 \mathrm{~g} / 100 \mathrm{~mL}$} \\
\hline & -Whey (1.96 g) & -Glucose $(0.7 \mathrm{~g})$ & -Saturated (0.64 g) & \\
\hline & -Casein $(7.84 \mathrm{~g})$ & -Lactose (3.4 g) & -MUFA (3.17 g) & \\
\hline & & -Isomaltulose (5.6g) & -PUFA (1.53 g) & \\
\hline & & -Polysaccharides (5.5g) & & \\
\hline \multirow{4}{*}{$\begin{array}{l}\text { High protein and low fat } \\
\text { (Almased) [16] }\end{array}$} & Total: $53 \%$ & Total: $31 \%$ & Total: $2 \%$ & \multirow{4}{*}{$0.4 \mathrm{~g} / 100 \mathrm{~mL}$} \\
\hline & -Soy protein $50 \%$ & -Glucose $30 \%$ & -Saturated (50\%) & \\
\hline & -Raw enzyme-rich bee honey $25 \%$ & & & \\
\hline & -Skim milk yogurt powder $23 \%$ & & & \\
\hline \multirow{5}{*}{$\begin{array}{l}\text { Composed of lactose, } \\
\text { isomaltulose, and resistant } \\
\text { starch [29] }\end{array}$} & Total: $19 \%$ & Total: $47 \%$ & Total: $32 \%$ & \multirow{5}{*}{$2 \mathrm{~g} / 100 \mathrm{~mL}$} \\
\hline & -Whey: $2.4 \mathrm{~g}$ & -Sugars: $11.3 \mathrm{~g}$ & (/100mL) & \\
\hline & -Soy: $2.4 \mathrm{~g}$ & -Lactose: $3.6 \mathrm{~g}$ & -Saturated: $0.5 \mathrm{~g}$ & \\
\hline & & & -MUFA: $2.2 \mathrm{~g}$ & \\
\hline & & & -PUFA: $1.1 \mathrm{~g}$ & \\
\hline \multirow{4}{*}{$\begin{array}{l}\text { Novasource }{ }^{\circledast} \text { Diabet }[27] \\
(/ 100 \mathrm{~mL})\end{array}$} & \multirow{4}{*}{ Total: $4.6 \mathrm{~g}$} & \multirow{4}{*}{ Total: $12 \mathrm{~g}$} & Total: $3.8 \mathrm{~g}$ & \multirow{4}{*}{$1.7 \mathrm{~g} / 100 \mathrm{~mL}$} \\
\hline & & & -Saturated: $1.3 \mathrm{~g}$ & \\
\hline & & & -MUFA: $1.4 \mathrm{~g}$ & \\
\hline & & & -PUFA: $0.8 \mathrm{~g}$ & \\
\hline \multirow{4}{*}{ Nutren Diabetes [9] } & \multirow{4}{*}{ Total: $15 \%$} & \multirow{4}{*}{ Total: $45 \%$} & Total: $40 \%$ & \multirow{4}{*}{$15.3 / 1000 \mathrm{kcal}$} \\
\hline & & & -Saturated $4 \%$ & \\
\hline & & & -MUFA: $26 \%$ & \\
\hline & & & -PUFA: 8\% (TEI) & \\
\hline \multirow{3}{*}{ Ultra-Glucose Control [10] } & \multirow{3}{*}{ Total: $15 \mathrm{~g}$} & \multirow{3}{*}{ Total: $27 \mathrm{~g}$} & Total: 7g & \multirow{3}{*}{$3 g / 56 g$} \\
\hline & & & -MUFA:4.5 g & \\
\hline & & & -Saturated: $1 \mathrm{~g}$ & \\
\hline \multirow{4}{*}{ Standard Formula** [27] } & \multirow{4}{*}{ Total: $4 \mathrm{~g}$} & Total: $13.6 \mathrm{~g}$ & Total: $3.3 \mathrm{~g}$ & \multirow{4}{*}{ N/A } \\
\hline & & -Sugars: $0.4 \mathrm{~g}$ & -Saturated fat: $1.3 \mathrm{~g}$ & \\
\hline & & -Lactose: $<0.01$ & -MUFA: $1.4 \mathrm{~g}$ & \\
\hline & & & -PUFA: 0.8 & \\
\hline
\end{tabular}

MUFA: monounsaturated fatty acid, PUFA: polyunsaturated fatty acid, N/A: not available

*There are no diabetes $1 / 2$ specific formulas

**Brand name not specified 
Table 2: Summary of studies evaluating DSF as meal replacement.

\begin{tabular}{|c|c|c|c|c|c|c|}
\hline Title & Objective & Population & Study Designs & $\begin{array}{c}\text { Formula } \\
\text { Composition }\end{array}$ & $\begin{array}{l}\text { Measured } \\
\text { Variables }\end{array}$ & Outcomes \\
\hline $\begin{array}{l}\text { Randomised } \\
\text { controlled trial of } \\
\text { four commercial } \\
\text { weight loss } \\
\text { programmes in the } \\
\text { UK: initial findings } \\
\text { from the BBC "diet } \\
\text { trials" (2006) }\end{array}$ & $\begin{array}{l}\text { To compare the } \\
\text { effectiveness of four } \\
\text { commercial weight } \\
\text { loss diets available to } \\
\text { adults in the United } \\
\text { Kingdom }\end{array}$ & $\begin{array}{l}210 \text { individuals who } \\
\text { were aged between } \\
18 \text { and } 65 \text { and had a } \\
\text { self-reported body } \\
\text { mass index between } \\
27 \text { and } 40 .\end{array}$ & $\begin{array}{l}\text { A multi-center, open- } \\
\text { label, randomized } \\
\text { controlled study. } \\
\text { Interventions Dr Atkins' } \\
\text { new diet revolution, } \\
\text { Slim-Fast plan, Weight } \\
\text { Watchers pure points } \\
\text { programme, and } \\
\text { Rosemary Conley's eat } \\
\text { yourself slim diet and } \\
\text { fitness plan. } \\
\text { TIME FRAME: } 6 \text { months }\end{array}$ & $\mathrm{N} / \mathrm{S}$ & $\begin{array}{l}\text { Weight reduction, } \\
\text { fat loss, age, } \\
\text { BMI, waist } \\
\text { circumference, } \\
\text { blood pressure, } \\
\text { glucose, total } \\
\text { cholesterol, } \\
\text { compliance and } \\
\text { withdrawal. }\end{array}$ & $\begin{array}{l}\text {-After six months all diets } \\
\text { resulted in a clinically useful } \\
\text { mean } \\
\text {-Body weight reduction: } \\
\text { Rosemary Conley } 9.9 \% \\
\text { (SD 5.6\%), Weight Watchers } \\
9.0 \%(5.6 \%) \text {, Atkins } 8.9 \%(5.6 \%) \text {, } \\
\text { and Slim-Fast } 6.8 \%(5.3 \%) ; \\
\text {-Regression analysis showed } \\
\text { that total } \\
\text { Weight loss over time had the } \\
\text { greatest influence on systolic } \\
\text { and diastolic pressure (adjusted } \\
\text { R2 } 0.61 \text { for change in systolic } \\
\text { pressure and 0.79 for change in } \\
\text { diastolic pressure). } \\
\text {-Weight Watchers group was } \\
\text { fasting glucose significantly } \\
\text { lower than in the control group. }\end{array}$ \\
\hline $\begin{array}{l}\text { Effect of a } \\
\text { nutritional liquid } \\
\text { supplement } \\
\text { designed for the } \\
\text { patient } \\
\text { with diabetes } \\
\text { mellitus (Glucerna } \\
\text { SR) on the } \\
\text { postprandial } \\
\text { glucose state, } \\
\text { insulin secretion } \\
\text { and insulin } \\
\text { sensitivity in } \\
\text { healthy } \\
\text { subjects } \\
\text { (2006) }\end{array}$ & $\begin{array}{l}\text { Identify the effect of } \\
\text { a nutritional liquid } \\
\text { supplement designed } \\
\text { for the patient with } \\
\text { diabetes mellitus } \\
\text { (Glucerna SR) in } \\
\text { single administration } \\
\text { on the postprandial } \\
\text { glucose state, insulin } \\
\text { secretion and insulin } \\
\text { sensitivity in healthy } \\
\text { subjects. }\end{array}$ & $\begin{array}{l}14 \text { non-obese } \\
\text { volunteers. Subjects } \\
\text { received a single } \\
\text { administration of } \\
300 \mathrm{kcal} \text {, gauged } \\
\text { with water at } 350 \\
\mathrm{ml} \text {, of each of } \\
\text { the following (at } \\
\text { least } 3 \text { days apart) } \\
\text { Glucerna; Ensure. }\end{array}$ & $\begin{array}{l}\text { A randomized, single- } \\
\text { blind, cross-over, clinical } \\
\text { trial. } \\
\text { TIME FRAME: } 1 \text { day }\end{array}$ & \begin{tabular}{|} 
Ensure high \\
calcium provides \\
0.95 kcal, $54.7 \%$ \\
carbohydrates, \\
$21.3 \%$ proteins \\
and $24.0 \%$ fat. On \\
the other hand, \\
each milliliter of \\
Glucerna SR supplies \\
0.93 kcal, $47.2 \%$ \\
carbohydrates, \\
$20.0 \%$ proteins and \\
$32.8 \%$ fat.
\end{tabular} & $\begin{array}{l}\text { Glucose level, } \\
\text { insulin, total } \\
\text { cholesterol, } \\
\text { high-density } \\
\text { lipoprotein and } \\
\text { low-density } \\
\text { lipoprotein } \\
\text { cholesterol, } \\
\text { triglycerides, } \\
\text { creatinine, and } \\
\text { uric acid, was } \\
\text { measured. }\end{array}$ & $\begin{array}{c}\text {-Glucose level at } 120 \text { min was } \\
\text { significantly lower after receiving } \\
\text { Ensure high calcium or Glucerna } \\
\text { SR compared to glucose } 75 \mathrm{~g} \\
\text {-Total insulin secretion was } \\
\text { significantly reduced after } \\
\text { Glucerna SR, with a statistic } \\
\text { tendency to be lower in its first } \\
\text { phase ( } p=0.07 \text { ). } \\
\text {-Insulin sensitivity was increased } \\
\text { with Glucerna SR }\end{array}$ \\
\hline $\begin{array}{l}\text { Nutrient adequacy } \\
\text { during weight loss } \\
\text { interventions: } \\
\text { a randomized } \\
\text { study in women } \\
\text { comparing the } \\
\text { dietary intake in a } \\
\text { meal replacement } \\
\text { group with a } \\
\text { traditional food } \\
\text { group. } \\
\text { (2007) }\end{array}$ & $\begin{array}{l}\text { To determine } \\
\text { and analyze the } \\
\text { nutritional adequacy } \\
\text { of a traditional } \\
\text { food-group diet } \\
\text { intervention } \\
\text { compared to } \\
\text { a traditional } \\
\text { food-group diet } \\
\text { intervention that also } \\
\text { incorporated meal } \\
\text { replacements as a } \\
\text { strategy for weight } \\
\text { loss. }\end{array}$ & $\begin{array}{c}96 \text { healthy } \\
\text { overweight / obese } \\
\text { women aged } \\
\text { between } 25-50 .\end{array}$ & $\begin{array}{l}\text { A randomized, cross } \\
\text { over, controlled trial. } \\
\text { Traditional Food Group } \\
\text { or a Meal Replacement } \\
\text { group, the latter } \\
\text { incorporated } 1-2 \text { meal } \\
\text { replacement bars o } \\
\text { drinks each day. } \\
\text { TIME FRAME: } 1 \text { year }\end{array}$ & $\begin{array}{c}\text { Protein 7-10g } \\
\text { CHO } 40-46 \text { g Fat } \\
1.5-3 g \\
\text { Fibre 5g Energy } 220 \\
\text { kcal }\end{array}$ & $\begin{array}{l}\text { Weight loss, body } \\
\text { fat, weight, waist } \\
\text { circumference, } \\
\text { resting energy } \\
\text { expenditure. }\end{array}$ & $\begin{array}{l}\text {-Mean weight loss was not } \\
\text { significantly different between } \\
\text { the two groups. } \\
\text {-Mean intake of meal } \\
\text { replacements was lower than } \\
\text { recommended. }\end{array}$ \\
\hline $\begin{array}{l}\text { Administration of } \\
\text { a new diabetes- } \\
\text { specific enteral } \\
\text { formula results in } \\
\text { an improved } 24 \\
\text { h glucose profile } \\
\text { in type2 diabetic } \\
\text { patients } \\
\text { (2009) }\end{array}$ & $\begin{array}{l}\text { To study the effect of } \\
\text { several boluses of a } \\
\text { new diabetes-specific } \\
\text { formula (DSF) during } \\
\text { the day on } 24 \mathrm{~h} \\
\text { glucose profile. }\end{array}$ & $\begin{array}{l}12 \text { diabetic type } \\
\text { subjects with a } \\
\text { mean age of } 67 \\
\text { years. }\end{array}$ & $\begin{array}{l}\text { A randomized, } \\
\text { controlled, double- } \\
\text { blind, cross-over study } \\
\text { in Netherlands. Group } \\
\text { I first received the } \\
\text { diabetes-specific formula } \\
\text { followed by the standard } \\
\text { formula and group II } \\
\text { received the formulas in } \\
\text { opposite order. } \\
\text { TIME FRAME: } 24 \mathrm{~h}\end{array}$ & $\begin{array}{c}\text { Energy Kcal (ml)393 } \\
\pm 47 \\
\text { Protein }(\mathrm{g}) 19.3 \pm 2.3 \\
\text { CHO (g) } 45.6 \pm 5.5 \\
\text { Fat (g) } 14.9 \pm 1.8 \\
\text { Fibres (g) } 7.9 \pm 0.9\end{array}$ & $\begin{array}{c}\text { Glycemia, insulin, } \\
\text { glucagon }\end{array}$ & $\begin{array}{l}\text {-Fasting glucose levels were not } \\
\text { significantly different among the } \\
\text { two groups. } \\
\text {-Mean glucose level was } \\
\text { significantly lower in the } \\
\text { diabetes-specific formula group } \\
\text { during the total } 24 \mathrm{~h} \text {. } \\
\text {-Diabetes specific formula } \\
\text { administration provided a } 26 \% \\
\text { reduction of total hyperglycemic } \\
\text { time (>10 mmol/L) over } 24 \mathrm{~h} \\
\text { compared with the standard } \\
\text { formula }(7.52 .3 \mathrm{~h} \text { versus } 10.2 \\
2.0 \mathrm{~h}, \mathrm{p}<0.05) \text {. }\end{array}$ \\
\hline
\end{tabular}




\begin{tabular}{|c|c|c|c|c|c|c|}
\hline $\begin{array}{l}\text { Effects of } \\
\text { short-term } \\
\text { low- and high- } \\
\text { carbohydrate diets } \\
\text { on postprandial } \\
\text { metabolism in } \\
\text { non diabetic and } \\
\text { diabetic subjects } \\
\text { (2009) }\end{array}$ & $\begin{array}{l}\text { To clarify whether } \\
\text { the apparently } \\
\text { adverse metabolic } \\
\text { effects of high- } \\
\text { carbohydrate diets, } \\
\text { at least in the short- } \\
\text { term, are dependent } \\
\text { upon the nature of } \\
\text { the carbohydrate } \\
\text { that replaces the fat. }\end{array}$ & $\begin{array}{l}\text { Eight healthy non- } \\
\text { diabetic subjects. } \\
\text { Ages ranging from } \\
40-61 \text { years. }\end{array}$ & $\begin{array}{l}\text { A randomized cross-over } \\
\text { study consisting of a } \\
\text { short-term, intensive } \\
\text { dietary modification. } \\
\text { TIME FRAME: Short- } \\
\text { term }\end{array}$ & \begin{tabular}{|c|} 
High-fat: \\
$-50 \%$ fat- $15 \%$ \\
protein-35\% $\mathrm{CHO}$ \\
High-starch: \\
$-15 \%$ fat-15\% protein \\
$-70 \%$ CHO. \\
High-sugar: \\
$-15 \%$ fat $-15 \%$ \\
protein- $70 \%$ CHO.
\end{tabular} & $\begin{array}{l}\text { Plasma glucose, } \\
\text { insulin, plasma } \\
\text { triacylglycerol, } \\
\text { non-esterified } \\
\text { fatty acids }\end{array}$ & $\begin{array}{l}\text {-Fasting tryacilglycerol } \\
\text { concentrations were greatest } \\
\text { following the high-sugar diet } \\
\text { (mean SEM for all subjects } \\
1900420 \text { mmol/I) and lowest } \\
\text { following high-fat (1010130 } \\
\text { mmol/I) }(\mathrm{P}=0.001 \text { ); high-starch } \\
\text { (mean } 1500310 \text { ) and high-fat did } \\
\text { not differ significantly ( } \mathrm{P}=0.06 \text { ). } \\
\text {-Fasting glucose concentrations } \\
\text { were not affected by prior } \\
\text { diet, but postprandial glucose } \\
\text { concentrations were }(\mathrm{P}=0.018 \text { ), } \\
\text { with significantly higher values } \\
\text { after the high-fat than the high- } \\
\text { sugar diet }(\mathrm{P}=0.03 \text { ). }\end{array}$ \\
\hline $\begin{array}{l}\text { Effect of a low- } \\
\text { calorie high } \\
\text { nutritional value } \\
\text { formula on weight } \\
\text { loss in type } 2 \\
\text { diabetes mellitus } \\
\text { (2010) }\end{array}$ & $\begin{array}{l}\text { Demonstrate that } \\
\text { substitution of one } \\
\text { of the main meals } \\
\text { with a low-calorie } \\
\text { diabetes-specific } \\
\text { meal replacement } \\
\text { could improve the } \\
\text { weight loss without } \\
\text { interfering negatively } \\
\text { with the nutritional } \\
\text { status of the obese } \\
\text { type } 2 \text { diabetic } \\
\text { subjects. }\end{array}$ & $\begin{array}{c}96 \text { obese diabetic } \\
\text { subjects (BMI } \\
33-44 \mathrm{~kg} / \mathrm{m}^{2} \text { ) }\end{array}$ & $\begin{array}{c}\text { An open label, non } \\
\text { randomized, cross-over } \\
\text { intervention. } \\
\text { TIME FRAME: } 24 \text { weeks }\end{array}$ & \begin{tabular}{|} 
Protein (g) $9.0(20 \%$ \\
kcal). \\
Fat (g) $6.0(33 \%$ \\
kcal). \\
CHO (g) $25.0(47 \%$ \\
kcal)
\end{tabular} & $\begin{array}{l}\text { Body weight, } \\
\text { HbA1c, plasma } \\
\text { glucose, insulin, } \\
\text { iron, iron- } \\
\text { binding capacity, } \\
\text { hemogram, } \\
\text { protein } \\
\text { electrophoresis } \\
\text { and lipids }\end{array}$ & $\begin{array}{l}\text {-The standard deviation of blood } \\
\text { glucose mean was reduced by } \\
50 \% \text { in the intervention group } \\
\text { but did not change in the control } \\
\text { group. } \\
\text {-The diastolic blood pressure } \\
\text { difference did not reach } \\
\text { statistical significance in the } \\
\text { intervention group }\end{array}$ \\
\hline $\begin{array}{l}\text { Effects of a } \\
\text { diabetes-specific } \\
\text { enteral nutrition } \\
\text { on nutritional and } \\
\text { immune status of } \\
\text { diabetic, obese, } \\
\text { and endotoxemic } \\
\text { rats: Interest of a } \\
\text { graded arginine } \\
\text { supply (2012) }\end{array}$ & \begin{tabular}{|} 
To investigate the \\
effects of a diabetes- \\
specific diet enriched \\
or not with arginine in \\
a model of infectious \\
stress in a diabetes \\
and obesity situation. \\
As a large intake \\
of arginine may be \\
deleterious, this \\
amino acid was given \\
in graded fashion.
\end{tabular} & $\begin{array}{l}22 \text { eleven-week- } \\
\text { old male ZDF (fa/ } \\
\text { fa) rats, obtained } \\
\text { from Charles River } \\
\text { Laboratories (Saint- } \\
\text { Germain sur l' } \\
\text { Abresle, France). }\end{array}$ & $\begin{array}{l}\text { A randomized controlled } \\
\text { experimental study } \\
\text { where diabetic rats } \\
\text { were administered } \\
\text { intraperitoneal } \\
\text { lipopolysaccharide } \\
\text { and fed with either a } \\
\text { diabetes-specific formula } \\
\text { (two variations } 1,2 \text { ) or } \\
\text { with graded arginine } \\
\text { supply, or a standard } \\
\text { nutrition. } \\
\text { TIME FRAME: } 7 \text { days }\end{array}$ & \begin{tabular}{|} 
Formula HP G \\
-Energy (kcal) 500500 \\
-Proteins (g) 2519 \\
-Fat (g)19.322 \\
-Saturated fatty \\
acids (g) \\
9.33 \\
MUFAs (g)3.7 16 \\
PUFAs (g) 43 \\
-CHO (g) 56.755 \\
Saccharose (g) 6.7 \\
$<0.5$ \\
Starch (g) 5055 \\
-Fibers (g) 0.1 7.5 \\
Soluble (\%)N/A 75 \\
Insoluble (\%)N/A 25
\end{tabular} & $\begin{array}{l}\text { Plasma glucose, } \\
\text { tal cholesterol, } \\
\text { tryglicerides, } \\
\text { insulin, individual } \\
\text { free amino acids, } \\
\text { plasma proteins. }\end{array}$ & $\begin{array}{l}\text {-Survival rate was higher in the } \\
\text { G group. } \\
\text {-All animals lost weight, but } \\
\text { the HP group weight loss was } \\
\text { more pronounced, although not } \\
\text { statiscally significant. (GA: } 4.2 \\
\pm 1.4 \mathrm{~g}, \mathrm{G:}: 4.5 \pm 0.8 \mathrm{~g}, \mathrm{HP}: 8.0 \\
\pm 1.2 \mathrm{~g} \text { ). }\end{array}$ \\
\hline $\begin{array}{l}\text { Meal } \\
\text { Replacements for } \\
\text { Weight Loss in } \\
\text { Type } 2 \text { Diabetes } \\
\text { in a Community } \\
\text { Setting (2012) }\end{array}$ & $\begin{array}{c}\text { Evaluate the use of } \\
\text { MR compared with } \\
\text { a diet book for } 6 \\
\text { months }\end{array}$ & $\begin{array}{l}120 \text { overweight and } \\
\text { obese subjects } \\
\text { with type } 2 \text { diabetes } \\
\text { mellitus were } \\
\text { recruited. Inclusion } \\
\text { criteria were type } \\
2 \text { diabetes (HbA1c } \\
6.5-12 \% \text { ), age } 20-65 \\
\text { years, not greater } \\
\text { than } 140 \mathrm{~kg}\end{array}$ & $\begin{array}{c}\text { A open label, } \\
\text { randomized, controlled } \\
\text { intervention. Subjects } \\
\text { in the intervention were } \\
\text { advised to consume } 2 \\
\text { MR/day for 3months and } \\
1 \mathrm{MR} / \text { day } \\
\text { for } 3 \text { months and follow } \\
\text { the manufacturers' } \\
\text { instructions from } \\
\text { printed material and the } \\
\text { website. Subjects in the } \\
\text { control arm were given } \\
\text { a commercially available } \\
\text { diet book. } \\
\text { TIME FRAME: } 6 \text { months }\end{array}$ & $\mathrm{N} / \mathrm{S}$ & $\begin{array}{l}\text { Weight loss, } \\
\text { HbA1c, serum } \\
\text { lipids, plasma } \\
\text { glucose and } \\
\text { insulin. }\end{array}$ & $\begin{array}{c}\text {-Consumption of } 2 \mathrm{MR} \text { for } \\
3 \text { months and } 1 \mathrm{MR} \text { for the } \\
\text { subsequent } 3 \text { months led to } \\
\text { weight loss of } 5.5 \mathrm{~kg}(5 \%) \\
\text { while the diet book group had } \\
\text { a weight loss of } 3 \mathrm{~kg}(3 \%)(\mathrm{P}= \\
0.027) \text {. } \\
\text {-Decreases in HbA1c were } 0.22 \% \\
\text { and } 0.12 \% \text {, for intervention and } \\
\text { control group respectively. } \\
\text {-Weight loss at } 6 \text { months was } 3.4 \\
\text { kg in MR and } 1.8 \mathrm{~kg} \text { in control } \\
(\mathrm{P}=0.07) \text {. }\end{array}$ \\
\hline $\begin{array}{l}\text { Respuesta } \\
\text { glucémica e } \\
\text { insulinémica a dos } \\
\text { fórmulas enterales } \\
\text { isocalóricas en } \\
\text { pacientes con } \\
\text { diabetes mellitus } \\
\text { tipo } 2 \text { (2013) }\end{array}$ & $\begin{array}{l}\text { To compare glycemic } \\
\text { and insulinemic } \\
\text { response on type } \\
2 \text { diabetes patients } \\
\text { after administration } \\
\text { of either a diabtes- } \\
\text { specific formula or a } \\
\text { standard formula. }\end{array}$ & $\begin{array}{c}15 \text { diabetes type } \\
2 \text { patients whose } \\
\text { HbA } 1 \mathrm{C}<9,0 \% \text {. }\end{array}$ & $\begin{array}{l}\text { A cross-over randomized } \\
\text { study where patients } \\
\text { received either a } \\
\text { diabetes-specific formula } \\
\text { or a standar isocaloric } \\
\text { formula. TIME FRAME: } \\
2 \text { weeks }\end{array}$ & $\begin{array}{l}\text { Protein Total: } 4.6 \mathrm{~g} \\
\text { CHO Total: } 12 \mathrm{~g} \text { Fat } \\
\text { Total: } 3.8 \mathrm{~g} \\
\text {-Saturated: } 1.3 \mathrm{~g} \\
\text {-MUFA: } 1.4 \mathrm{~g} \\
\text {-PUFA: } 0.8 \mathrm{~g} \text { Fibre } \\
\text { Total: } 1.7 \mathrm{~g} / 100 \mathrm{~mL}\end{array}$ & Glycemia, insulin & $\begin{array}{l}\text {-Insulinemia was lower in the } \\
\text { disbetes-specific formula during } \\
\text { the length of the study. } 4,39 \mu \mathrm{U} / \\
\mathrm{ml}(\mathrm{IC} 95 \%: 0,927 \text { a } 7,87) \\
\text {-Glycemia levels were lower in } \\
\text { the diabetes-specific formula at } \\
60,90,120,150,180 \text { minutes } \\
\text { than the standard formula } \\
\text { group. }\end{array}$ \\
\hline
\end{tabular}




\begin{tabular}{|c|c|c|c|c|c|c|}
\hline $\begin{array}{l}\text { Meal replacement } \\
\text { reduces insulin } \\
\text { requirement, } \\
\text { HbA1c and weight } \\
\text { long-term in type } \\
2 \text { diabetes patients } \\
\text { with >100 U insulin } \\
\text { per day } \\
(2013)\end{array}$ & \begin{tabular}{|c|} 
Examine whether \\
an energy-restricted \\
protein-rich meal \\
replacement (PRMR) \\
can help to reduce the \\
insulin requirement \\
in patients with type \\
2 diabetes using \\
$>100$ insulin per day \\
and simultaneously \\
improve HbA1c, \\
weight and other \\
cardiometabolic risk \\
factors.
\end{tabular} & $\begin{array}{l}22 \text { patients with } \\
\text { type } 2 \text { diabetes, } \\
\text { body mass index } \\
\text { (BMI) }>27 \text {, age } 35- \\
75 \text { years, insulin } \\
\text { therapy with }>100 \mathrm{U} \\
\text { insulin per day. }\end{array}$ & $\begin{array}{l}\text { An open label, clinical } \\
\text { trial, non-randomized. } \\
\text { TIME FRAME: } 12 \text { weeks }\end{array}$ & $\begin{array}{c}\text { Energy } 360 \mathrm{kcal} \\
\text { Protein }(\%) 53.4 \\
\text { Fat }(\%) 2.0 \\
\text { CHO (\%)30.6 } \\
\text { Fibres }(\mathrm{g}) 0.4\end{array}$ & $\begin{array}{c}\text { Insulin } \\
\text { requirement, } \\
\text { HbA1C, weight } \\
\text { loss, body mass } \\
\text { index, blood lipid } \\
\text { levels. }\end{array}$ & $\begin{array}{c}\text {-Mean insulin dose was reduced } \\
\text { from } 147(75) \cup \text { to } 65(32) \cup(P< \\
0.0001) \text { after } 12 \text { weeks of study. } \\
\text {-HbA1c decreased from } 8.8 \% \\
(1.4 \%) \text { to } 8.1 \%(1.6 \%)(P=0.048) \\
\text {-Weight decreased from } 118.0 \\
\text { (19.7) kg to } 107.4(19.2) \mathrm{kg}(P< \\
0.0001) .\end{array}$ \\
\hline $\begin{array}{l}\text { Blood Glucose and } \\
\text { Insulin Responses } \\
\text { to Two Hypocaloric } \\
\text { Enteral Formulasin } \\
\text { Patients with } \\
\text { Diabetes Mellitus } \\
\text { Type } 2 \\
\text { (2013) }\end{array}$ & $\begin{array}{c}\text { Compare the } \\
\text { glycaemic and } \\
\text { insulinemic response } \\
\text { of type } 2 \text { diabetic } \\
\text { patients after oral } \\
\text { administration of } 250 \\
\text { ml of two enteral } \\
\text { formulas: a specific } \\
\text { formula (Nova source } \\
{ }^{\circledR} \text { Diabet Smartflex }{ }^{\circledR} \text { ) } \\
\text { against a standard } \\
\text { isocaloric formula. }\end{array}$ & $\begin{array}{c}15 \text { patientes } \\
\text { with controlled } \\
\text { type } 2 \text { diabetes } \\
\text { mellitus(dietary } \\
\text { advice or oral } \\
\text { antidiabetics), } \\
\text { and aglycosylated } \\
\text { hemoglobin less } \\
\text { than } 9.0 \%\end{array}$ & $\begin{array}{c}\text { A cross-over, } \\
\text { randomized, controlled } \\
\text { trial. }\end{array}$ & $\begin{array}{l}\text { Energy103 kcal } \\
\text { Protein }(\mathrm{g}) 4,6 \\
\text { Fat }(\mathrm{g}) 3,8 \\
\mathrm{CHO}(\mathrm{g}) 12,0 \\
\text { Fibres }(\mathrm{g}) 1,7\end{array}$ & $\begin{array}{l}\text { Blood glucose, } \\
\text { insulin, weight, } \\
\text { BMI }\end{array}$ & $\begin{array}{c}\text {-Patients receiving DSF showed } \\
\text { a lower mean AUC0-t of glucose, } \\
\text { mean difference }-4,753.26 \mathrm{mg} / \\
\text { min/dl ( } 95 \% \mathrm{Cl}:-7,256.7 \text { to } \\
-2,249.82) \text {, } \\
\text { Intervention group showed mean } \\
\text { insulinemia significantly lower } \\
\text { AUC0-t, mean difference: } 930.27 \\
\text { uU/min/ml ( } 95 \% \mathrm{Cl}-1,696.34 \text { to } \\
-164.2) \text {. } \\
\text {-Analysis of glucose } \\
\text { concentrations in the total study } \\
\text { shows that the group with the } \\
\text { specific formula has a lower mean } \\
\text { glucose } 25.77 \text { mg / dl (95\% Cl } \\
\text { 18.29 to } 33.25 \text { ), the same fact was } \\
\text { detected with insulinemia } 4.39 \\
\text { mU/ml (95\% Cl: } 0.927 \text { to } 7.87 \text { ). }\end{array}$ \\
\hline $\begin{array}{l}\text { Taking a Low } \\
\text { Glycemic Index } \\
\text { Multi-Nutrient } \\
\text { Supplement as } \\
\text { Breakfast Improves } \\
\text { Glycemic Control } \\
\text { in Patients with } \\
\text { Type } 2 \text { Diabetes } \\
\text { Mellitus: A } \\
\text { Randomized } \\
\text { Controlled Trial } \\
\text { (2014) }\end{array}$ & $\begin{array}{l}\text { Examine the effect } \\
\text { of a low glycemic } \\
\text { index (GI) multi- } \\
\text { nutrient supplement, } \\
\text { consumed in place } \\
\text { of breakfast, on } \\
\text { glycemic control in } \\
\text { patients with type } \\
2 \text { diabetes mellitus } \\
\text { (T2DM). }\end{array}$ & $\begin{array}{l}\text { A total of } 71 \\
\text { participants were } \\
\text { randomized at } \\
\text { a 2:1 ratio into } \\
\text { either a breakfast } \\
\text { replacement } \\
\text { group or a normal } \\
\text { breakfast group } \\
\text { for a } 12 \text {-week } \\
\text { interventional study. }\end{array}$ & $\begin{array}{c}\text { A randomized, open } \\
\text { label, interventional } \\
\text { study on patients with } \\
\text { T2DM. } \\
\text { TIME FRAME: } 12 \text { weeks }\end{array}$ & $\begin{array}{c}\text { Energy Kcal } 404.9 \pm \\
180.0 \\
\text { Protein }(\mathrm{g}) 12.9 \pm 5.5 \\
\text { Fat }(\mathrm{g}) 10.3 \pm 7.9 \\
\text { CHO }(\mathrm{g}) 67.2 \pm 33.2 \\
\text { Fibres }(\mathrm{g}) 3.0 \pm 2.7\end{array}$ & $\begin{array}{l}\text { Age, Body Mass } \\
\text { Index, waist } \\
\text { circumference, } \\
\text { blood pressure, } \\
\text { physical activity, } \\
\text { fasting glucose } \\
\text { level, liver } \\
\text { function, renal } \\
\text { function, blood } \\
\text { lipid levels, blood } \\
\text { glucose levels and } \\
\text { HbA1c }\end{array}$ & $\begin{array}{l}\text {-The breakfast replacement group } \\
\text { had no significant increase in } \\
\text { fasting blood glucose (FBG) at } \\
\text { week } 12, \text { while the } \mathrm{FBG} \text { in control } \\
\text { group increased by } 1.4 \mathrm{mmol} / \mathrm{L} \\
(95 \% \mathrm{Cl} \text { for change, } 0.8 \text { to } 1.9 \\
\mathrm{mmol} / \mathrm{L}, \mathrm{p}<0.001) \text {. } \\
\text {-There was a statistically } \\
\text { significant difference in } \mathrm{BMI} \\
\text { between the treatment and } \\
\text { control groups ( } \mathrm{p}=0.032 \text { ) due } \\
\text { to the weight gain in the control } \\
\text { group (mean change was } 0.5 ; 95 \% \\
\mathrm{Cl} \text { was } 0.2 \text { to } 0.9, \mathrm{p}=0.007 \text { ). }\end{array}$ \\
\hline $\begin{array}{l}\text { The impact of a } \\
\text { weight reduction } \\
\text { program with and } \\
\text { without meal- } \\
\text { replacement on } \\
\text { health-related } \\
\text { quality of life in } \\
\text { middle-aged obese } \\
\text { females (2014) }\end{array}$ & \begin{tabular}{|} 
Assess the impact \\
of two 12-month \\
weight reduction \\
interventions (one \\
arm including a meal \\
replacement) on \\
changes in HRQOL \\
among obese \\
females.
\end{tabular} & $\begin{array}{c}31 \text { obese adults } \\
\text { (between } 30-41 \mathrm{~kg} / \\
\mathrm{m}^{2} \text { ) were recruited. } \\
\text { One group received } \\
\text { the weight-reduction } \\
\text { lifestyle program } \\
\text { without a meal } \\
\text { replacement (LS) } \\
\text { and the other group } \\
\text { received the same } \\
\text { lifestyle program } \\
\text { with the addition of } \\
\text { a soy-based meal } \\
\text { replacement product } \\
\text { (LSMR). }\end{array}$ & $\begin{array}{l}\text { An open label, non- } \\
\text { randomized, controlled } \\
\text { trial. }\end{array}$ & $\begin{array}{l}\text { Protein (\%)53.3 } \\
\text { Fat (\%)2 } \\
\text { CHO (\%) } 31 \\
\text { Fibres (g) } 0.4\end{array}$ & $\begin{array}{l}\text { Health-Related } \\
\text { Quality of Life } \\
\text { (SF-36), BMI, } \\
\text { total activity and } \\
\text { weight loss. }\end{array}$ & $\begin{array}{l}\text {-Females taking the meal } \\
\text { replacement product (LSMR) } \\
\text { reported lower baseline } \\
\text { HRQOL scores than the control } \\
\text { subgroup. This difference was } \\
\text { significant in six of eight HRQOL } \\
\text { dimensions and was most } \\
\text { pronounced in the scores for } \\
\text { vitality and health perception } \\
\text {-After } 12 \text { months of the } \\
\text { intervention, body weight was } \\
\text { reduced in both groups (LS: }-6.6 \\
\pm 6.6 p<0.001 \text { vs. LSMR-7.6 } \\
\pm 7.9 \text { kg; } p<0.001 \text { ). Weight } \\
\text { reduction was more pronounced } \\
\text { (p }=0.1 \text { ) in the females taking } \\
\text { the soy-based meal replacement } \\
\text { product. } \\
\text {-Lifestyle behavior expressed by } \\
\text { physical fitness (Watt/kg body } \\
\text { weight) and leisure time physical } \\
\text { activity (hours of physical } \\
\text { activity per week) increased } \\
\text { similarly in both groups }\end{array}$ \\
\hline
\end{tabular}




\begin{tabular}{|c|c|c|c|c|c|c|}
\hline $\begin{array}{l}\text { Effect of consuming } \\
\text { a formula with } \\
\text { carbohydrates. } \\
\text { prolonged release } \\
\text { on the glycemic } \\
\text { response and } \\
\text { insulin post- } \\
\text { prandial in healthy } \\
\text { individuals. } \\
\text { (2016) }\end{array}$ & $\begin{array}{l}\text { To determine the } \\
\text { glycemic response } \\
\text { and post-prandial } \\
\text { response of healthy } \\
\text { individuals when } \\
\text { they consumed an } \\
\text { extended-release } \\
\text { carbohydrate } \\
\text { formula. }\end{array}$ & $\begin{array}{c}21 \text { healthy subjects, } \\
11 \text { men and } 10 \\
\text { women. Ages } \\
\text { between } 17-25 \\
\text { years. }\end{array}$ & $\begin{array}{c}\text { A crossover study } \\
\text { where subjects went } \\
\text { through } 4 \text { different tests, } \\
\text { 1-weekinterval between } \\
\text { each test and type of } \\
\text { nutrition. } 2 \text { weeks for } \\
\text { the reference meal and } \\
2 \text { weeks for the enteral } \\
\text { formula. } \\
\text { TIME FRAME: } 4 \text { weeks }\end{array}$ & \begin{tabular}{|} 
Protein Total: $21 \%$ \\
-Calcium casein ate \\
$(98 \%)$ \\
-Soy protein (2\%) \\
CHO Total: $56 \%$ \\
Maltodextrin, \\
fructose and maltitol \\
Fat Total: $15 \%$ \\
Oleic acid and soy \\
oil. Fibre $3.6 \mathrm{~g} /$ \\
$100 \mathrm{~mL}$
\end{tabular} & $\begin{array}{l}\text { BMI, abdominal } \\
\text { circumference, } \\
\text { basal and post } \\
\text { pandrial insulin, } \\
\text { basal and post } \\
\text { pandrial glycemia, } \\
\text { cholesterol, } \\
\text { triglycerides. }\end{array}$ & $\begin{array}{l}\text {-Post prandial glycemia was } \\
\text { significantly lower when taking } \\
\text { the diabetes-specific formula. } \\
\text {-There was no significant } \\
\text { difference between the two } \\
\text { groups at 120min insulin } \\
\text { measurement. } \\
\text {-Area under the curve was } \\
\text { significantly smaller when } \\
\text { compared to the reference meal. }\end{array}$ \\
\hline $\begin{array}{l}\text { Structured lifestyle } \\
\text { intervention } \\
\text { based on a trans- } \\
\text { cultural diabetes- } \\
\text { specific nutrition } \\
\text { algorithm (tDNA) } \\
\text { in individuals with } \\
\text { type } 2 \text { diabetes: } \\
\text { a randomized } \\
\text { controlled trial } \\
\text { (2017) }\end{array}$ & \begin{tabular}{|} 
To evaluate the \\
effectiveness of \\
the trans-cultural \\
diabetes nutrition \\
algorithm \\
(tDNA) versus usual \\
Diabetes care in \\
primary care settings.
\end{tabular} & $\begin{array}{c}230 \text { subjects with } \\
\text { type } 2 \text { diabetes } \\
\text { with } \mathrm{HbA} 1 \mathrm{c} \text { levels } \\
7 \%-11 \% \text {, not treated } \\
\text { with insulin. }\end{array}$ & $\begin{array}{l}\text { A randomized, open- } \\
\text { label, clinical trial in } \\
\text { Malaysia. } \\
\text { TIME FRAME: } 1 \text { year }\end{array}$ & $\mathrm{N} / \mathrm{S}$ & $\begin{array}{l}\text { HbA1c, weight, } \\
\text { fasting plasma } \\
\text { glucose, systolic } \\
\text { blood pressure, } \\
\text { lipid profile, C } \\
\text { reactive protein. }\end{array}$ & $\begin{array}{c}\text {-The median values for FPG was } \\
\text { significantly }(\mathrm{p}<0.001) \text { lower } \\
\text { in the tDNA-MI patients ( } 6.9 \\
\pm 1.8 \mathrm{mmol} / \mathrm{L}) \text { compared with } \\
\text { tDNA-CC }(7.6 \pm 2.9 \mathrm{mmol} / \mathrm{L}) \text { and } \\
\text { UC }(7.8 \pm 2.7 \mathrm{mmol} / \mathrm{L}) \text {. } \\
\text {-The body weight and BMI } \\
\text { reduced significantly in both } \\
\text { the tDNA intervention groups, } \\
\text { where tDNA-MI group achieved } \\
\text { a greater (mean } \pm \text { SE) weight } \\
\text { loss of } 6.9 \pm 1.3 \mathrm{~kg}(\mathrm{p}<0.001) \text {. } \\
\text {-At } 6 \text { months, A1c decreased } \\
\text { significantly in tDNA-MI }(-1.1 \\
\pm 0.1 \%, \mathrm{p}<0.001) \text { and tDNA-CC } \\
(-0.5 \pm 0.1 \%, p=0.001) \text { but not in } \\
\text { UC }(-0.2 \pm 0.1 \%, p=N S) \text {. }\end{array}$ \\
\hline $\begin{array}{l}\text { Effects of meal } \\
\text { replacement } \\
\text { therapy } \\
\text { on metabolic } \\
\text { outcomes in Thai } \\
\text { patients } \\
\text { with type } 2 \\
\text { diabetes: A } \\
\text { randomized } \\
\text { controlled trial } \\
\text { (2018) }\end{array}$ & $\begin{array}{c}\text { To compare } \\
\text { metabolic outcomes } \\
\text { between T2DM } \\
\text { patients receiving } \\
\text { the new MR formula } \\
\text { (ONCE PRO) and } \\
\text { normal controlled } \\
\text { diets. }\end{array}$ & $\begin{array}{c}110 \text { diabetes } 2 \\
\text { patients mean age } \\
53 \text { years. }\end{array}$ & $\begin{array}{l}\text { A multi-center open } \\
\text { label randomized } \\
\text { controlled study in } \\
\text { Thailand .Subjects were } \\
\text { assigned to either a meal } \\
\text { replacement group or a } \\
\text { normal controlled diet. } \\
\text { TIME FRAME: } 10 \text { months }\end{array}$ & \begin{tabular}{|} 
Protein Total: $20 \%$ \\
-Soy protein isolate \\
(50\%) \\
-Whey protein isolate \\
$(50 \%)$ \\
CHO Total:40\% \\
-Maltodextrin (46.5\%) \\
-Isomaltulose (18.5\%) \\
-Maltitol (18.5\%) \\
-Fibersol (9.26\%) \\
-Fructooligosaccharide \\
$(4.62 \%)$ \\
Other (2.53\%) \\
Fat (MUFAs $23 \%$ of \\
total calories) \\
-Canola oil (48.8\%) \\
-High-oleic safflower \\
oil (24.4\%) \\
-Rice bran oil (22.0\%) \\
-Fish oil (4.87\%)
\end{tabular} & $\begin{array}{l}\text { HbA1c, fasting } \\
\text { plasma glucose, } \\
\text { lipid profile, waist } \\
\text { circumference, } \\
\text { body mass index, } \\
\text { systolic and } \\
\text { diastolic blood } \\
\text { pressure. }\end{array}$ & $\begin{array}{l}\text {-HbA1c reduction was only } \\
\text { statically significant on the } \\
\text { control group at the third month } \\
\text { from } 7.82+/ 0.61 \text { to } 7.55+/ \\
0.79, \mathrm{p}=0.001 . \\
\text {-LDL- C level increased in the } \\
\text { control group ( }+6.01 \mathrm{mg} / \mathrm{dL} \text { ) } \\
\text { and decreased in the meal } \\
\text { replacement group (-2.72 mg/ } \\
\mathrm{dL} \text { ). Difference between groups } \\
\text { was significant, ( } \mathrm{p}=0.049 \text { ). } \\
\text {-BMI decreased significantly in } \\
\text { both groups at the third month } \\
\text { however; there was no statistical } \\
\text { difference between groups at } \\
\text { the end of the intervention. }\end{array}$ \\
\hline $\begin{array}{l}\text { Individualized } \\
\text { Meal Replacement } \\
\text { Therapy Improves } \\
\text { Clinically Relevant } \\
\text { Long-Term } \\
\text { Glycemic Control in } \\
\text { Poorly Controlled } \\
\text { Type } 2 \text { Diabetes } \\
\text { Patients } \\
\text { (2018) }\end{array}$ & \begin{tabular}{|} 
Validate whether \\
individualized intense \\
meal replacement \\
by a low- \\
carbohydrate \\
formula diet with \\
accompanied self- \\
monitoring of blood \\
glucose (SMBG) \\
contributes to long- \\
term improvements \\
in HbA1c, weight, \\
and cardiometabolic \\
risk factors in poorly \\
controlled type 2 \\
diabetes.
\end{tabular} & $\begin{array}{l}409 \text { patients with } \\
\text { type } 2 \text { diabetes, } \\
\text { aged } 25-79 \\
\text { years with poorly } \\
\text { controlled glucose } \\
\text { levels (HbA1c } 7.5 \% \text { ), } \\
\text { and body mass index } \\
\text { (BMI) } 27 \mathrm{~kg} / \mathrm{m} 2 \\
\text { were included in the } \\
\text { study. }\end{array}$ & $\begin{array}{l}\text { A randomized, open } \\
\text { label, clinical trial, } \\
\text { parallel groups. Study } \\
\text { consisted of one } \\
\text { observational control } \\
\text { group and either } \\
\text { a moderate group } \\
\text { (M-group) with two meal } \\
\text { replacements/day or a } \\
\text { stringent group (S-group) } \\
\text { with three meal } \\
\text { replacements/day. } \\
\text { TIME FRAME: } 12 \text { weeks } \\
\text { of meal replacement } \\
\text { intervention and } 52 \\
\text { weeks of follow-up }\end{array}$ & $\begin{array}{c}\text { Energy360 kcal } \\
\text { Protein }(\%) 53.4 \\
\text { Fat }(\%) 2.0 \\
\text { CHO }(\%) 30.6 \\
\text { Fibres }(\mathrm{g}) 0.4\end{array}$ & $\begin{array}{l}\text { Sex, age weight, } \\
\text { BMI, HbA1c, } \\
\text { fasting blood } \\
\text { glucose, blood } \\
\text { pressure, blood } \\
\text { lipids, current } \\
\text { treatment. }\end{array}$ & $\begin{array}{l}\text {-Both intervention groups } \\
\text { achieved improvements in } \\
\text { HbA1c, fasting blood glucose, } \\
\text { blood pressure, and weight (all } p \\
<0.001 \text { ) within } 12 \text { weeks. } \\
\text {-S-group showed a clinically } \\
\text { relevant improvement in HbA1c } \\
\text { of }-0.81 \% \text { [-1.06; }-0.55] \text { ( } p< \\
0.001 \text { ) after } 52 \text { weeks of follow- } \\
\text { up, whereas HbA1c was not } \\
\text { statistically different between } \\
\text { the M- and control group }\end{array}$ \\
\hline
\end{tabular}




\begin{tabular}{|c|c|c|c|c|c|c|}
\hline $\begin{array}{l}\text { Change in } \\
\text { Cardiometabolic } \\
\text { Risk Factors } \\
\text { Associated With } \\
\text { Magnitude } \\
\text { of Weight Regain } 3 \\
\text { Years After a 1-Year } \\
\text { Intensive Lifestyle } \\
\text { Intervention in } \\
\text { Type } 2 \text { Diabetes } \\
\text { Mellitus: The Look } \\
\text { AHEAD Trial (2019) }\end{array}$ & $\begin{array}{c}\text { Induce a mean loss } \\
\geq 7 \% \text { of initial weight } \\
\text { and to increase } \\
\text { participants' } \\
\text { moderately intense } \\
\text { physical activity to } \geq \\
175 \text { minutes a week. }\end{array}$ & $\begin{array}{l}1561 \text { individuals } \\
\text { with a body mass } \\
\text { index }>25 \mathrm{~kg} / \mathrm{m} 2 \\
\text { (or }>27 \mathrm{~kg} / \mathrm{m} 2 \text { if } \\
\text { taking insulin) and } \\
\text { a type } 2 \text { diabetes } \\
\text { mellitus diagnosis }\end{array}$ & $\begin{array}{l}\text { A cross-over } \\
\text { randomized trial. } \\
\text { Lifestyle Intervention; } \\
\text { Group treatment } \\
\text { sessions during the } \\
\text { first year; liquid meal } \\
\text { replacements; and } \\
\text { optional weight loss } \\
\text { medication, after the } \\
\text { first } 6 \text { months, with } \\
\text { selected individuals. } \\
\text { TIME FRAME: 1-year } \\
\text { intensive lifestyle } \\
\text { intervention and with } \\
\text { year } 4 \text { follow-up data }\end{array}$ & $\begin{array}{c}\text { Four meal } \\
\text { replacements, } \\
\text { including: Slim Fast } \\
\text { (Slim Fast Foods), } \\
\text { Glucerna (Ross } \\
\text { Laboratories), } \\
\text { OPTIFAST (Novartis } \\
\text { Nutrition) and HMR } \\
\text { (HMR, Inc.). }\end{array}$ & $\begin{array}{l}\text { Weight loss, } \\
\text { weekly physical } \\
\text { activity, serum } \\
\text { lipids, blood } \\
\text { glucose, } \\
\text { hormones, } \\
\text { cardiovascular } \\
\text { morbidity, } \\
\text { medication and } \\
\text { mortality. }\end{array}$ & $\begin{array}{l}\text {-Participants with } \geq 10 \% \text { initial } \\
\text { weight loss had a significantly } \\
\text { lower proportion of participants } \\
\text { starting on diabetes mellitus } \\
\text { medication ( } 3.1 \% \text { versus } 7.5 \% \text { ) } \\
\text {-In men only, losing } \geq 10 \% \text { initial } \\
\text { weight was associated with a } \\
\text { significantly lower proportion } \\
\text { starting antihypertensive } \\
\text { medication ( } 15.5 \% \text { versus } 7.4 \% \text { ) } \\
\text {-Among men, at baseline, } \\
\text { those with }<10 \% \text { initial weight } \\
\text { loss had significantly higher } \\
\text { HbA1cconcentrations and } \\
\text { diastolic blood pressure than } \\
\text { those with } \geq 10 \% \text { initial weight } \\
\text { loss }\end{array}$ \\
\hline $\begin{array}{l}\text { Effect of Oral } \\
\text { Nutritional } \\
\text { Supplements } \\
\text { with Sucromalt } \\
\text { and Isomaltulose } \\
\text { versus Standard } \\
\text { Formula on } \\
\text { Glycaemic Index, } \\
\text { Entero-Insular } \\
\text { Axis Peptides and } \\
\text { Subjective Appetite } \\
\text { in Patients with } \\
\text { Type } 2 \text { Diabetes: A } \\
\text { Randomised Cross- } \\
\text { Over Study (2019) }\end{array}$ & $\begin{array}{l}\text { Assess sucromalt/ } \\
\text { isomaltulose ONS-D } \\
\text { effects on the } \\
\text { glycaemic } \\
\text { response (GI/GL), } \\
\text { EIAP release and } \\
\text { postprandial SA } \\
\text { in type } 2 \text { diabetic } \\
\text { individuals }\end{array}$ & $\begin{array}{l}23 \text { DM2 subjects } \\
\text { over } 50 \text { years old; } \\
\text { Body mass index } \\
\text { (BMI) between } 18.5 \\
\mathrm{~kg} / \mathrm{m} 2-35 \mathrm{~kg} / \mathrm{m}\end{array}$ & $\begin{array}{l}\text { A randomized, double- } \\
\text { blind, cross-over } \\
\text { study. In this study, } \\
\text { three oral nutritional } \\
\text { supplements were } \\
\text { examined: non-diabetes- } \\
\text { specific standard oral } \\
\text { nutritional supplements } \\
\text { (ET; Ensure); oral } \\
\text { supplements with a } \\
\text { blend of slow-digesting } \\
\text { carbohydrates including } \\
\text { resistant maltodextrin } \\
\text { and sucromalt (GS; } \\
\text { Glucerna) and oral } \\
\text { supplements composed } \\
\text { of lactose, isomaltulose, } \\
\text { and resistant starch (DI; } \\
\text { Dia sip) } \\
\text { TIME FRAME: } 1 \text { day }\end{array}$ & $\begin{array}{c}\text { Composition* } \\
\text { ET, DI GS } \\
\text { Calories (kcal) } \\
10510493 \\
\text { Protein (g) 3.84.94.3 } \\
\text { Fat (g) 2.53.83.5 } \\
\text { Carbohydrate (g) } \\
17.311 .710 .9 \\
\text { Dietary Fibre } \\
\text { (g)1.02.0 1.8 } \\
\text { *ET(Ensure); GS } \\
\text { (Glucerna); DI } \\
\text { (Diasip) }\end{array}$ & $\begin{array}{l}\text { Plasma insuline, } \\
\text { BMI, weight, } \\
\text { blood glucose, } \\
\text { HbA1c, Plasma } \\
\text { total GIP, GLP-1, } \\
\text { total cholesterol, } \\
\text { triacylglycerides, } \\
\text { LDL-C and HDL- } \\
\text { Clevels. }\end{array}$ & $\begin{array}{l}\text {-At } 150 \text { min, ET presented a } \\
\text { higher glucose concentration } \\
\text { than DSF ( } p<0.001) \text {, but no } \\
\text { significant differences were } \\
\text { found in insulin concentration } \\
\text { between DI and GS ( } p=0.976 \text { ). } \\
\text { At } 150 \text { min, value of this incretin } \\
\text { was significantly higher for GS } \\
\text { when compared with both, ET ( } p \\
<0.001 \text { ) and DI ( } p<0.001 \text { ). } \\
\text {-The AUC0-180 min in insulin } \\
\text { response was significantly lower } \\
\text { in GS when compared with the } \\
\text { other supplements ( } p<0.001 \text { ) } \\
\text {-The maximum peak of this } \\
\text { incretin was observed at } 90 \\
\text { min with ET and DI, which was } \\
\text { higher when compared to GS } \\
\text { levels ( }<<0.05 \text { ). }\end{array}$ \\
\hline
\end{tabular}

specific formulas for diabetics have been developed in the last decade. With the aim of being used with multidisciplinary weight management programs as meal replacements [28]. DSF provide a controlled caloric intake, minimizing postprandial glucose response; even in cases where patients are resistant to traditional weight loss therapy, adding the approach of meal replacements with these formulas has proven to be effective, improving weight loss to $-5.4 \mathrm{~kg}$ in some studies $[18,23]$.

Meanwhile standard formulas are well known and have been used over the past few decades, the used of DSFs is still controversial. Diverse studies have shown the important role of DSF for type-2 diabetic patients management in fields such as glycemic control (both capillary and plasma glucose), lowering insulin requirements, effective weight loss as well as reducing the risk of acquired infections in hospitalized patients [32]. Compared to standard formulas, DSF have lower glycemic index and its components are targeting a population who needs normoprotein-caloric diet [34]. An ideal nutritional formula for all types of diabetic patients has not yet been developed. For this reason, integrated therapy remains the best option [30]. One of the elements, exercise, is an irreplaceable component to achieve long-term maintenance of lost weight and reduce the risk of deaths from cardiovascular diseases in diabetics. The goal of achieving up to $10 \%$ of weight loss in obese diabetic patients brings long-term benefits such as reducing the need for hypoglycemic agents and better glycemic control [21]

On the other hand, there are still many aspects of topic pending for investigation, including comparison of the effects of protein-rich and fat-rich DSF on short-term and long-term. In addition, the scientific community still lacks enough clinical trials to investigate the impact of different therapies on morbidity and mortality among diabetic adults [20].

\section{References}

1. World Health Organization (2019) Diabetes.

2. Long AN, Dagogo-Jack S (2011) Comorbidities of diabetes and hypertension: mechanisms and approach to target organ protection. J Clin Hypertens (Greenwich) 13: 244-251.

3. CDC (2020) Estimates of Diabetes and Its Burden in the United States. National Diabetes Statistics Report.

4. Chawla A, Chawla R, Jaggi S (2016) Microvasular and macrovascular complications in diabetes mellitus: Distinct or continuum? Indian J Endocrinol Metab 20: 546-551.

5. Elia M, Ceriello A, Laube H, Sinclair AJ, Engfer M, et al. (2005) Enteral nutritional support and use of diabetes-specific formulas for patients with diabetes: a systematic review and meta-analysis. Diabetes Care 28: 2267-2679.

6. Sanz-Paris A, Boj-Carceller D, Lardies-Sanchez B, Perez-Fernandez L, Cruz-Jentoft AJ (2016) Health-Care Costs, Glycemic Control and Nutritional Status in Malnourished Older Diabetics Treated with a Hypercaloric Diabetes-Specific Enteral Nutritional Formula. Nutrients 8: 153. 
7. Angarita L, López J, Aparicio D, Parra K, Uzcátegui M, et al. (2016) Efecto del consumo de una fórmula con carbohidratos de liberación prolongada sobre la respuesta glicémica e insulina post-prandial en individuos sanos. ALAN 66: 113-120.

8. Hofman Z, De Van Drunen J, Kuipers H (2006) The Glycemic Index of standard and diabetes-specific enteral formulas. Asia Pac J Clin Nutr 15: 412-417.

9. Gulati S, Misra A, Nanda K, Pandey RM, Garg V, et al. (2015) Efficacy and tolerance of a diabetes specific formula in patients with type 2 diabetes mellitus: An open label, randomized, crossover study. Diabetes Metab Syndr 9: 252-257.

10. Mottalib A, Mohd-Yusof BN, Shehabeldin M, Pober DM, Mitri J, et al. (2016) Impact of Diabetes-Specific Nutritional Formulas versus Oatmeal on Postprandial Glucose, Insulin, GLP-1 and Postprandial Lipidemia. Nutrients 8 pii: E443.

11. Laksir H, Lansink M, Regueme SC, de Vogel-van den Bosch J, Pfeiffer $\mathrm{AFH}$, et al. (2018) Glycaemic response after intake of a high energy, high protein, diabetes-specific formula in older malnourished or at risk of malnutrition type 2 diabetes patients. Clin Nutr 37: 20842090.

12. Huhmann MB, Yamamoto S, Neutel JM, Cohen SS, Ochoa Gautier JB (2018) Very high-protein and low-carbohydrate enteral nutrition formula and plasma glucose control in adults with type 2 diabetes mellitus: a randomized crossover trial. Nutr Diabetes 8: 45.

13. Hofman Z, van Drunen JD, de Later C, Kuipers H (2004) The effect of different nutritional feeds on the postprandial glucose response in healthy volunteers and patients with type II diabetes. Eur J Clin Nutr 58: 1553-1556.

14. Wadden TA, West DS, Delahanty L, Jakicic J, Rejeski J, et al. (2006) The Look AHEAD study: a description of the lifestyle intervention and the evidence supporting it. Obesity (Silver Spring) 14: 737-752.

15. Li D, Zhang P, Guo H, Ling W (2014) Taking a low glycemic index multi-nutrient supplement as breakfast improves glycemic control in patients with type 2 diabetes mellitus: a randomized controlled trial. Nutrients 6: 5740-5755.

16. Kempf K, Schloot NC, Gärtner B, Keil R, Schadewaldt P, et al. (2014) Meal replacement reduces insulin requirement, $\mathrm{HbA1c}$ and weight long-term in type 2 diabetes patients with $>100 \mathrm{U}$ insulin per day. $J$ Hum Nutr Diet 27 Suppl 2: 21-27.

17. Kempf K, Röhling M, Niedermeier K, Gärtner B, Martin S (2018) Individualized Meal Replacement Therapy Improves Clinically Relevant Long-Term Glycemic Control in Poorly Controlled Type 2 Diabetes Patients. Nutrients 10 pii: E1022.

18. Keogh JB, Clifton PM (2012) Meal replacements for weight loss in type 2 diabetes in a community setting. J Nutr Metab 2012: 918571

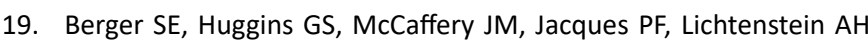
(2019) Change in Cardiometabolic Risk Factors Associated With Magnitude of Weight Regain 3 Years After a 1-Year Intensive Lifestyle Intervention in Type 2 Diabetes Mellitus: The Look AHEAD Trial. J Am Heart Assoc 8: e010951.

20. Kwok CF, Ho LT (2014) Look Action for Health in Diabetes trial: What we have learned in terms of real world practice and clinical trials. J Diabetes Investig 5: 637-638.
21. Wadden TA, West DS, Neiberg RH, Wing RR, Ryan DH, et al. (2009) One-year weight losses in the Look AHEAD study: factors associated with success. Obesity (Silver Spring) 17: 713-722.

22. Truby H, Baic S, deLooy A, Fox KR, Livingstone MB, et al. (2006) Randomised controlled trial of four commercial weight loss programmes in the UK: initial findings from the BBC "diet trials". BMJ 332: 1309-1314.

23. Tatti P, di Mauro P, Neri M, Pipicelli G, Mussad VA (2010) Effect of a low-calorie high nutritional value formula on weight loss in type 2 diabetes mellitus. Mediterr J Nutr Metab 3: 65-69.

24. Patankar N, Patankar N, Shah P (2016) Effect of balanced low calorie meal replacement formula diet in the management of overweight/ obesity and metabolic profile-a retrospective clinical experience report. Adv Obes Weight Manag Control 4: 166-170.

25. Koohkan S, McCarthy DH, Berg A (2017) The effect of a soy-yoghurthoney product on excess weight and related health risk factors-A review. J Nutrition Health Food Sci 5: 1-10.

26. Koohkan S, Schaffner D, Milliron BJ, Frey I, König D, et al. (2014) The impact of a weight reduction program with and without mealreplacement on health related quality of life in middle-aged obese females. BMC Womens Health 14: 45.

27. DA de Luis, O Izaola, B de la Fuente, K Araújo (2013) Respuesta glucémica e insulinémica a dos fórmulas entérales isocalóricas en pacientes con diabetes mellitus tipo 2. Nut Hosp 28: 600-606.

28. González-Ortiz M, Martínez-Abundis E, Hernández-Salazar E, KamRamos AM, Robles-Cervantes JA (2006) Effect of a nutritional liquid supplement designed for the patient with diabetes mellitus (Glucerna SR) on the postprandial glucose state, insulin secretion and insulin sensitivity in healthy subjects. Diabetes Obes Metab 8: 331-335.

29. Angarita Dávila L, Bermúdez V, Aparicio D, Céspedes V, Escobar MC, et al. (2019) Effect of Oral Nutritional Supplements with Sucromalt and Isomaltulose versus Standard Formula on Glycaemic Index, Entero-Insular Axis Peptides and Subjective Appetite in Patients with Type 2 Diabetes: A Randomised Cross-Over Study. Nutrients 11 pii: E1477.

30. Jhonson R (2016) Calorie Replacements: A Useful Tool to Support a Diabetes Weight Management Plan.

31. Mesejo A, Montejo-González JC, Vaquerizo-Alonso C, Lobo-Tamer G, Zabarte-Martinez M, et al. (2015) Diabetes-specific enteral nutrition formula in hyperglycemic, mechanically ventilated, critically ill patients: a prospective, open-label, blind-randomized, multicenter study. Crit Care 19: 390.

32. Olveira-Fuster G, Gonzalo-Marín M (2005) Fórmulas de nutrición enteral para personas con diabetes mellitus. Endocrinol Nutr 52: 516-524.

33. Mechanick JI, Marchetti AE, Apovian C, Benchimol AK, Bisschop PH, et al. (2012) Diabetes-specific nutrition algorithm: a transcultural program to optimize diabetes and prediabetes care. Curr Diab Rep 12: 180-194.

34. Doola R, Todd AS, Forbes JM, Deane AM, Presneill JJ, et al. (2018) Diabetes-Specific Formulae Versus Standard Formulae as Entera Nutrition to Treat Hyperglycemia in Critically III Patients: Protocol for a Randomized Controlled Feasibility Trial. JMIR Res Protoc 7: e90. 